\title{
Editorial
}

\section{Thanks to authors, reviewers and readers}

\section{Microbes and Infectious Diseases Editorial Board Members}

After successful release of the first 3 issues of Microbes and Infectious Diseases in 2020, we've received 105 submissions from different countries (Africa: Egypt, Sudan, Tunisia, Senegal, Nigeria, Cameroon, Gabon, and Benin; Asia: Saudi Arabia, India, Pakistan, Bangladesh, Hong Kong, and China; Europe: Bosnia and Herzegovina, Turkey, Austria, and Italy; Latin America: Cuba; and Australia). Although many of our papers are still in the peer review process, we have already published 55 papers online with a 36 $\%$ rejection rate. The median time from submission to online publication is 38 days.

The Editorial Board Members have worked hard to meet the goals of Microbes and Infectious Diseases in delivering timely decisions and maintaining publication of high-quality papers through vigorous and scientific peer review process. Besides, we believe that authors' response to the valuable comments of our editors and reviewers is a cornerstone for impacting in the international research community.

We would like to introduce our deep thanks to 68 individual reviewers who give so freely of their time and expertise to critically evaluating manuscripts for the journal. Our reviewers, like our authors, come from all over the world to maintain a wide authorship, readership and global citation activity. It is noteworthy that within the first 6 months of our journal's age, authors and reviewers are representing over 20 countries.

The policies and guidelines of Microbes and Infectious Diseases would help secure sufficient quality and novelty of content to become competitive with our national, regional and international competitors.

We would also like to take this opportunity to acknowledge our reviewers more publicly, highlighting our most outstanding reviewers. Those reviewers who not only carry out many assignments but also demonstrate professionalism and provide comments that truly help the authors improve their work.

\section{Reviewers List (in alphabetical order):}

$\begin{array}{lr}\text { Abeer Nafee } & \text { Egypt } \\ \text { Ahmed Asaad } & \text { Egypt } \\ \text { Ahmed Cihad } & \text { Turkey } \\ \text { Ajay Prajapati } & \text { India } \\ \text { Ali Alnasser } & \text { Saudi Arabia } \\ \text { Amal algendy } & \text { Egypt } \\ \text { Amal Atta } & \text { Egypt } \\ \text { Amani El Kholy } & \text { Egypt }\end{array}$




\begin{tabular}{|c|c|c|c|}
\hline Ayman Allam & Egypt & Nahed Mostafa & Egypt \\
\hline Edmond PUCA & Albania & Neveen antouny & Egypt \\
\hline Eman Elbehedy & Egypt & Olaniyan Mathew & Nigeria \\
\hline Faiza Amber Siddiqui & USA & Omar Atrooz & Jordan \\
\hline Fatma Abd El-Aziz & Egypt & Raghda Hafez & Egypt \\
\hline Fatma Moharram & Egypt & Rania Kishk & Egypt \\
\hline Ghada Mahmoud M. Fathy & Egypt & Rashad Abdul-Ghani & Yemen \\
\hline Hani Mowafi & Egypt & Reem Refat & Egypt \\
\hline Hosam Abd Elaziz & Egypt & Rehab El-Sokkary & Egypt \\
\hline Ibrahim Ogunyinka & Nigeria & Rooh Ullah & Pakistan \\
\hline James Oyeniyi & Nigeria & Sadek Refaat & Egypt \\
\hline Jihan Shawky & Egypt & Safaa Elnaggar & Egypt \\
\hline Khaled Aboshanab & Egypt & Said Abbadi & Egy \\
\hline Laila Alkady & Egypt & Samar El-Beshbishi & Egypt \\
\hline MabrouK Ghonaim & Egypt & Samia Etewa & Egypt \\
\hline Mahmoud Tawfick & Egypt & Sara Abdelrahman & Egy \\
\hline Mai Maamoun & Egypt & Shoukry Mahmoud & Egypt \\
\hline Makwin makut & Nigeria & Shahenda Badran & Egypt \\
\hline Manal Bahgat & Egypt & Shamshul Ansari & Nepal \\
\hline Matthew Okon & Nigeria & Soma Ajlan & Egypt \\
\hline Maysa Abdallah Saeed & Egypt & Tadesse Eguale & Ethiopia \\
\hline Mervat Hesham & Egypt & Takwa Farid & Egypt \\
\hline Moawia Elbadri & Sudan & Umar Abdullahi & Nigeria \\
\hline Mohammad Azizur & Bangladesh & Youssef Aboelwan & Egypt \\
\hline Mohammad Basharat & Pakistan & Wael Abdalazem & Egypt \\
\hline Mohamed ElFeky & Egypt & Wafaa Mosallamy & Egy \\
\hline Mohamed Al sweify & Egypt & Wafaa Zahran & Egy \\
\hline Mohamed Ansar & India & Walaa Khater & Egy \\
\hline Mohamed Sarhan & Egypt & Walid Faisal & Egypt \\
\hline Mohsen Hassan & Egypt & & \\
\hline Mona Aboserea & Egypt & & \\
\hline
\end{tabular}

